\title{
Examination of the Influence of Flight Altitude and Speed on the Efimtsov Model Parameters
}

\author{
Stefan Haxter* and Carsten Spehr ${ }^{\dagger}$ \\ German Aerospace Center DLR, D-37073 Göttingen, Germany
}

\begin{abstract}
A flight test was conducted and the data was evaluated in order to investigate the influence of flight altitude and flight speed on the coherence length model by Efimtsov. An underestimation of the measured values by the model was found below a frequency of $1 \mathrm{kHz}$. Above this frequency, the predicted values matched the measured values very well. The Efimtsov ansatz was found to be suitable for the description of coherence length, when the parameters are adapted. Three further analyses were performed: the flight speed was varied with constant altitude, the altitude was varied with constant flight speed, and both speed \& altitude were varied together. No considerable variation was measured when the flight speed was varied separately. A small variation was determined at low frequency when the flight altitude was altered separately. When varying the parameters together, a distinct variation was found for the cases of highest speed and altitude.

The applicability of the exponential coherence decay was analyzed. The absolute deviation was found to increase with lower frequency. The characteristic of the deviation was found to collapse in different regions with different scaling of the strouhal number.
\end{abstract}

\section{Nomenclature}

$c_{f} \quad$ friction coefficient

C correction factor

$f \quad$ frequency, $\mathrm{Hz}$

$g \quad$ weighting of standard deviation input

$\Delta f$ frequency resolution, $\mathrm{Hz}$

$i$ index of FFT frequency node

$K$ angular wavenumber $\mathrm{m}^{-1}$

$l_{x} \quad$ coherence length, in-flow direction

$l_{y} \quad$ coherence length, cross-flow direction

$M \quad$ Mach number

$N \quad$ number of spacing combinations

$q$ dynamic pressure, $\mathrm{Pa}$

$u_{c} \quad$ convective velocity

$u_{0}$ free-stream velocity

$u_{\tau}$ friction velocity

$x \quad$ aircraft longitudinal direction, $\mathrm{m}$

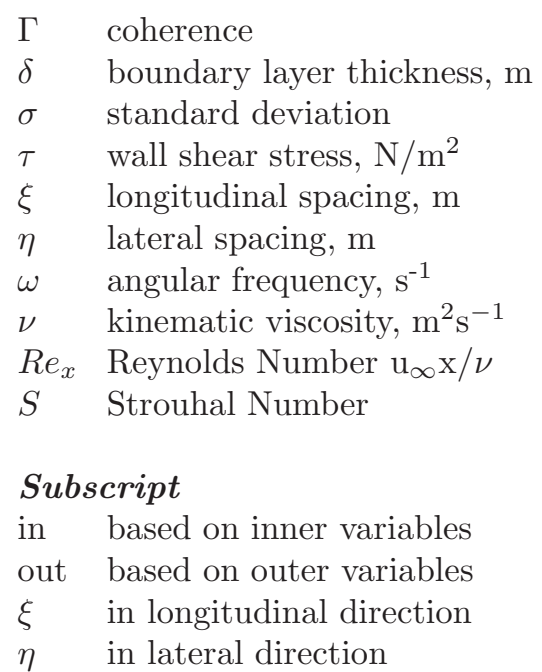

\section{Introduction}

In the past, several cross-spectral models have been set up to describe the excitation of the fuselage by the turbulent boundary layer empirically in order to predict the turbulent boundary layer (TBL)-portion

\footnotetext{
${ }^{*} \mathrm{PhD}$ student, Institute of Aerodynamics and Flow Technology - Experimental Methods, stefan.haxter@dlr.de, member AIAA

${ }^{\dagger}$ Research Engineer, Institute of Aerodynamics and Flow Technology - Experimental Methods, carsten.spehr@dlr.de, member AIAA
} 
of the cabin noise. The Efimtsov model ${ }^{1}$ is an enhancement of the preceding Corcos model. ${ }^{2}$ It takes into account a turbulent structure size limitation due to the boundary layer thickness. In contrast to the Corcos model, the Efimtsov model has been derived from flight test data rather than low-speed wind tunnel data and therefore is considered to be more applicable for the prediction of aircraft hull excitation. ${ }^{3}$ However, no information was found in the Efimtsov documentation concerning measurement equipment, positioning of the transducers on the test carrier, and flight attitude utilized for the setup of the model. Tests by Palumbo have shown that considerable deviation of the measured coherence length from the Efimtsov model may occur. ${ }^{4}$ In this article data from a flight test is discussed in order to examine the influence of flight speed and flight altitude on the governing parameters of the Efimtsov model. The setup is described by Spehr et al. ${ }^{5}$ and by Haxter \& Spehr. ${ }^{6}$

\section{Measurement Setup}

Flight test data was acquired using the DLR's model A320 Advanced Technology Research Aircraft (ATRA) in May of 2011. Three aluminum dummy windows were installed in the vicinity of the right hand side wing in three consecutively window banks. The run length from the tip of the aircraft nose to the center of the center dummy window was approximately $12.6 \mathrm{~m}$. A total number of 30 Kulites were installed in a quasi-randomized pattern that provided one neighbor element at the same longitudinal (aircraft length axis) and lateral (perpendicular to aircraft length axis on the surface) position. 435 different transducer spacings resulted from the distribution. The sampling frequency was $50 \mathrm{kHz}$.

$$
\frac{\delta}{x}=C \cdot 0.37 \cdot \operatorname{Re}_{x}^{-1 / 5} \cdot\left[1+\left(\frac{\operatorname{Re}_{x}}{6.9 \times 10^{7}}\right)^{2}\right]^{0.1}
$$

The boundary layer thickness $\delta$ was estimated using a formula provided by Bies $^{7}$ for high Reynolds numbers $R e_{x}$, where $x$ is the run length of the boundary layer. In this investigation, the formula was adapted to the condition of a jet airliner by a correction factor $C$. This factor is derived from measurement data provided by Gyorgyfalvy ${ }^{8}$ who conducted boundary layer thickness measurements on a Boeing 720 Jetliner (single aisle). The position of their boundary layer measurement was comparable to the position in the present investigation. The estimated thickness was scaled by the correction factor $C=1.1969$ to match the value measured by Gyorgyfalvy. The resulting formula (1) was then used to estimate the boundary layer thickness $\delta$ in the present investigation.

Gyorgyfalvy also provided an estimation of the surface friction coefficient $c_{f}=1.35 \times 10^{-3}$, which is used later on in this evaluation for the setup of the Efimtsov model.

A pressure gradient was present in the measurement region due to the vicinity of the wing. ${ }^{6}$

\section{Test Environment}

Three variations of speed and altitude were investigated: First, the speed was varied at constant altitude. Secondly, the altitude was varied at constant speed. Thirdly, both altitude and speed were varied combined.

A first evaluation at a single flight attitude at $T A S=236 \mathrm{~m} \mathrm{~s}^{-1}(M=0.78)$ and an altitude of $35,000 \mathrm{ft}$ was used to investigate methods for the determination of the coherence lengths (reference 6). The methods were now applied to other flight attitudes summarized in table 1. The flight level is abbreviated by "FL" and the true airspeed is abbreviated by "TAS". The variation is shown graphically in figure 1 . The temperature distribution at the different altitudes is shown in figure 2. The second highest flight attitude of the variation of both, temperature and speed stands out a little, since it deviates from a linear temperature decrease. The altitude of the tropopause, which is indicated by a constant temperature with change of altitude, seems to have shifted on the measurement days as indicated by the deviation of the highest flight level (FL390) to the ICAO standard atmosphere.

\section{Data Processing}

Each of the sensor pairings time-domain data was transformed into the frequency domain by using a Fast Fourier transform routine with $2^{13}$ sample points. The resulting spectra had a frequency resolution of $\Delta f=12.2 \mathrm{~Hz}$ and were combined and then averaged to provide the cross spectrum of the pairing. In order 


\begin{tabular}{|c|c|c|c|c|c|}
\hline FL (100ft) & \multicolumn{5}{|c|}{ TAS (m/s) } \\
\hline 250 & 212.0 & 224.8 & 232.1 & 235.8 & 244.9 \\
270 & 214.0 & 226.7 & & & \\
310 & 213.9 & & 231.6 & & \\
350 & 214.0 & & & 235.6 & \\
390 & 206.2 & & & & 236.1 \\
\hline
\end{tabular}

Table 1. Selected flight conditions

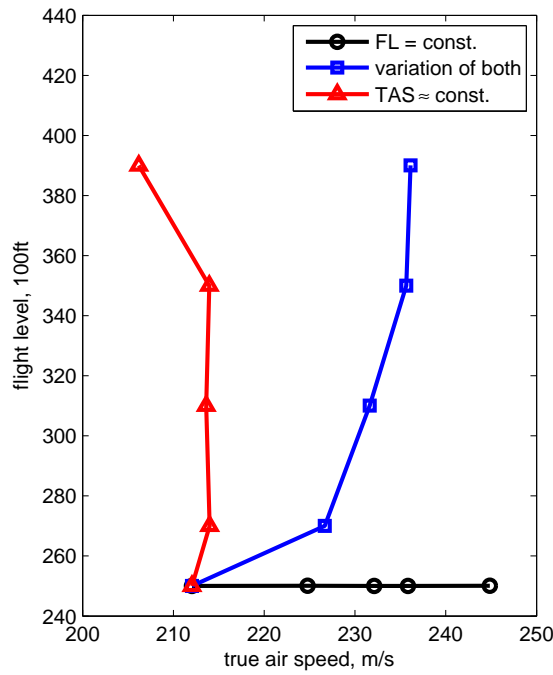

Figure 1. Plot of the variations

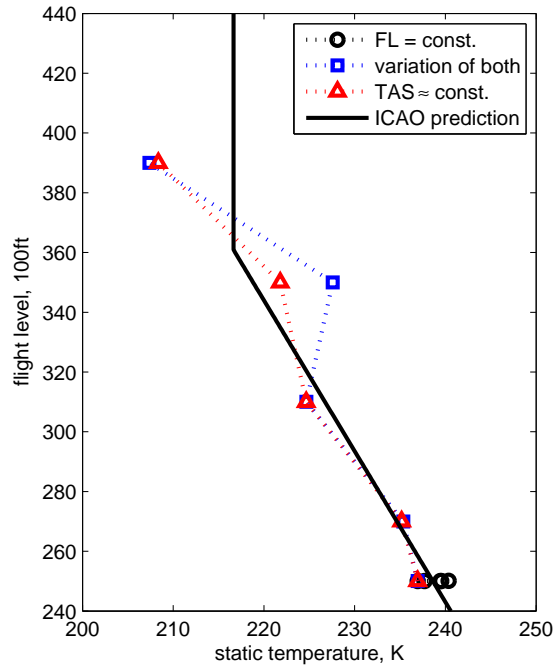

Figure 2. Outside Temperature at measurement points

to obtain the coherence spectra of the sensor pairs, the absolute values of the cross spectra were normalized with the auto spectra of the involved transducer signals. The resulting coherence over the spacing domain is shown for a single frequency of $f=928 \mathrm{~Hz}$ in figure 3 . The position of the spacing combinations in the measurements are shown as dots. Each dot provides a measured coherence value as measured by equation (2). $R$ denotes the cross spectral density of transducer signals $n$ and $m$.

$$
\Gamma_{n, m, \text { meas }}(f)=\frac{\left|R_{n, m}\right|}{\sqrt{R_{n, n} \cdot R_{m, m}}}
$$

A two-dimensional exponential fit can be applied to the experimental data points in order to determine the spatial decay of the self-similarity of coherent structures in the flow in longitudinal $(\xi)$ and lateral $(\eta)$ direction. ${ }^{9}$ This was done by Palumbo for one-dimensional decay. ${ }^{4}$ As suggested by Corcos, ${ }^{2}$ an exponential trial function is used.

$$
\Gamma_{f i t}(f)=\exp \left(-\frac{\xi}{l_{x}(f)}\right) \cdot \exp \left(-\frac{\eta}{l_{y}(f)}\right)
$$

The second dimension is added by a multiplicative approach used by Efimtsov and Corcos (equation (3)). The exponential fit provides a decay coefficient which can be used to describe the decay of self-similarity of coherent structures with a single parameter. Since the fit is two-dimensional, it provides a decay coefficient for both longitudinal and lateral direction - the values $l_{x, i}$ for the frequency index $i$. The decay coefficient is called the "coherence length".

The flow angle deviates locally from the aircraft longitudinal axis at the array location. Therefore, the orientation of the two dimensions of the fit is adapted to the local flow direction. The flow direction was 


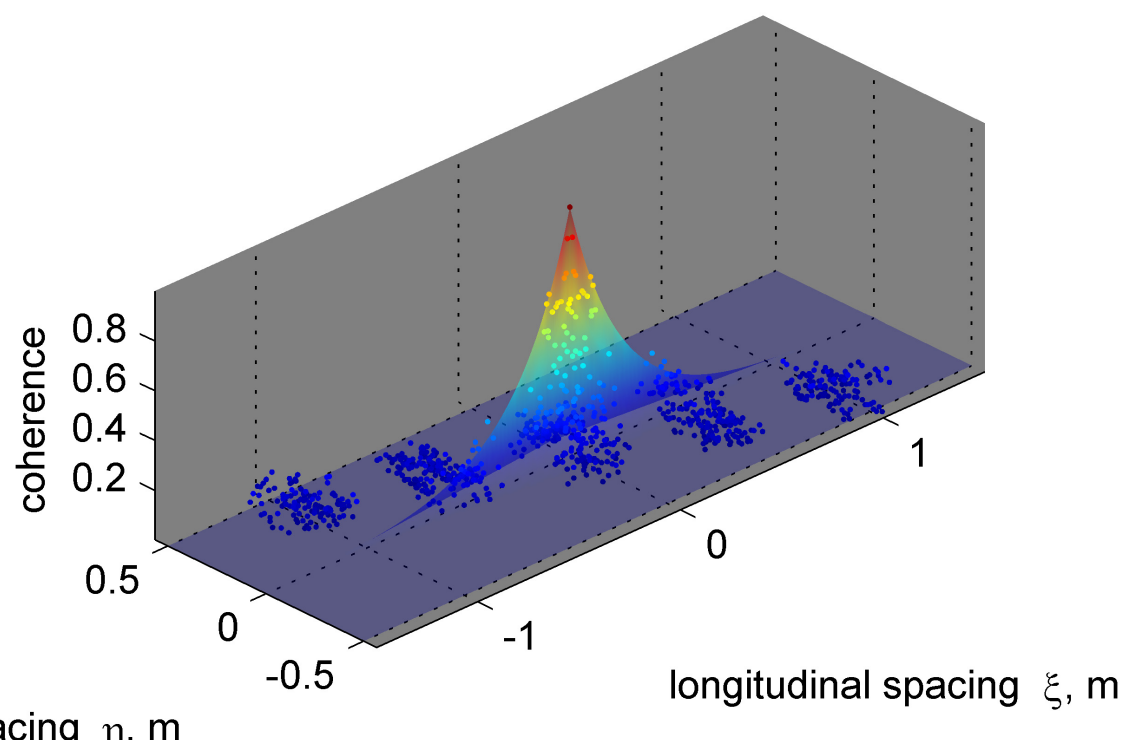

lateral spacing $\eta, \mathrm{m}$

Figure 3. example of the two-dimensional coherence fit at $f=928 \mathrm{~Hz}$

taken as the direction in which the coherence level between two transducer signals was above the threshold over the longest distance. A singular value decomposition was used to determine this direction. All spacing combinations with a coherence level above a threshold of $1 / e=0.368$ were taken into account. For frequencies above $2500 \mathrm{~Hz}$, this threshold was lowered to $1 / e^{2}=0.1353$ to avoid an error resulting from the limited spatial array resolution at small spacings.

The 2D-fit is applied to the coherence at all frequency nodes resulting from the Fourier Transform. The resulting experimental data points for the coherence length can be approximated by Efimtsov's model function (equation (4)). Besides the Strouhal number based on boundary layer thickness and friction velocity, $S_{\delta}$, the convective velocity $u_{c}(f)$, and the friction velocity $u_{\tau}$, this trial function has three characteristic parameters - $a_{1}, a_{2}, a_{3}$ - that can be extracted from the experimental coherence length data.

$$
l_{x}\left(S_{\delta}\right)=\left[\left(\frac{S_{\delta} \cdot a_{1}}{u_{c} / u_{\tau}}\right)^{2}+\frac{a_{2}^{2}}{S_{\delta}^{2}+\left(a_{2} / a_{3}\right)}\right]^{-\frac{1}{2}}
$$

The combination of boundary layer thickness $\delta$ and friction velocity $u_{\tau}$ as characteristic values for the Strouhal number will be referred to as "outer" scaling in the following, since the boundary layer thickness is a macroscopic flow parameter. The combination of kinematic viscosity $\nu$ and the square of the friction velocity $u_{\tau}^{2}$ is referred to as "inner" scaling in the following, since the kinematic viscosity flow parameter connected with viscous effects that are dominant in the viscous sub-layer.

The convective velocity in equation (4) was identified from the data by applying a wavenumber transformation on the cross spectra and extracting the maximum position of the convective ridge in the wavenumber domain. The wavenumber of the maximum corresponded with the convective velocity via

$$
u_{c}=\frac{\omega}{K}
$$

where $\omega$ is the angular frequency and $K$ is the angular wavenumber at which the maximum of the convective ridge occurs. Details about the calculation can be found in Haxter \& Spehr. ${ }^{6}$ The friction velocity $u_{\tau}$ is approximated using the friction coefficient determined by Gyorgyfalvy in at comparable flight attitude and position on a similar single-aisle jet airliner.

$$
u_{\tau}=c_{f} \cdot q
$$

The goodness of the fit was determined by the standard deviation between the fit and the measured data (equation (7)). In order to estimate the standard deviation for each direction separately, the deviation between fitted and measured coherence was weighted with the fitted coherence in the respective other 
direction.

$$
\sigma=\sqrt{\frac{1}{N-1} \sum_{i=1}^{N} g_{m} \cdot\left(\Gamma_{\text {meas }}-\Gamma_{f i t}\right)^{2}}
$$

In equation (7), $g_{m}$ is a weighting factor described by equations (8) and (9). Its function is to estimate the influence of the longitudinal and lateral direction on the standard deviation separately. For instance: in order to estimate the standard deviation resulting from the longitudinal direction, all spacing combinations that are positioned on the line $\eta=0$ are weighted "1" $\left(\exp \left((\eta=0) / l_{y}\right)\right)$ for the estimation. The further the spacing departs from the $\eta=0$ - axis, the less its influence on the standard deviation in the respective direction under consideration is. This weighting parameter $w_{m}$ is shown for an exemplary coherence length of $l_{x}=0.3 \mathrm{~m}$ in figure 4 and $l_{y}=0.05 \mathrm{~m}$ in figure 5 .

$m$ is the index for the respective direction $-\xi$ (longitudinal) or $\eta$ (lateral). The weighting factor is an estimation of how far - in terms of distance - the influence of turbulent structures in the boundary layer extend.

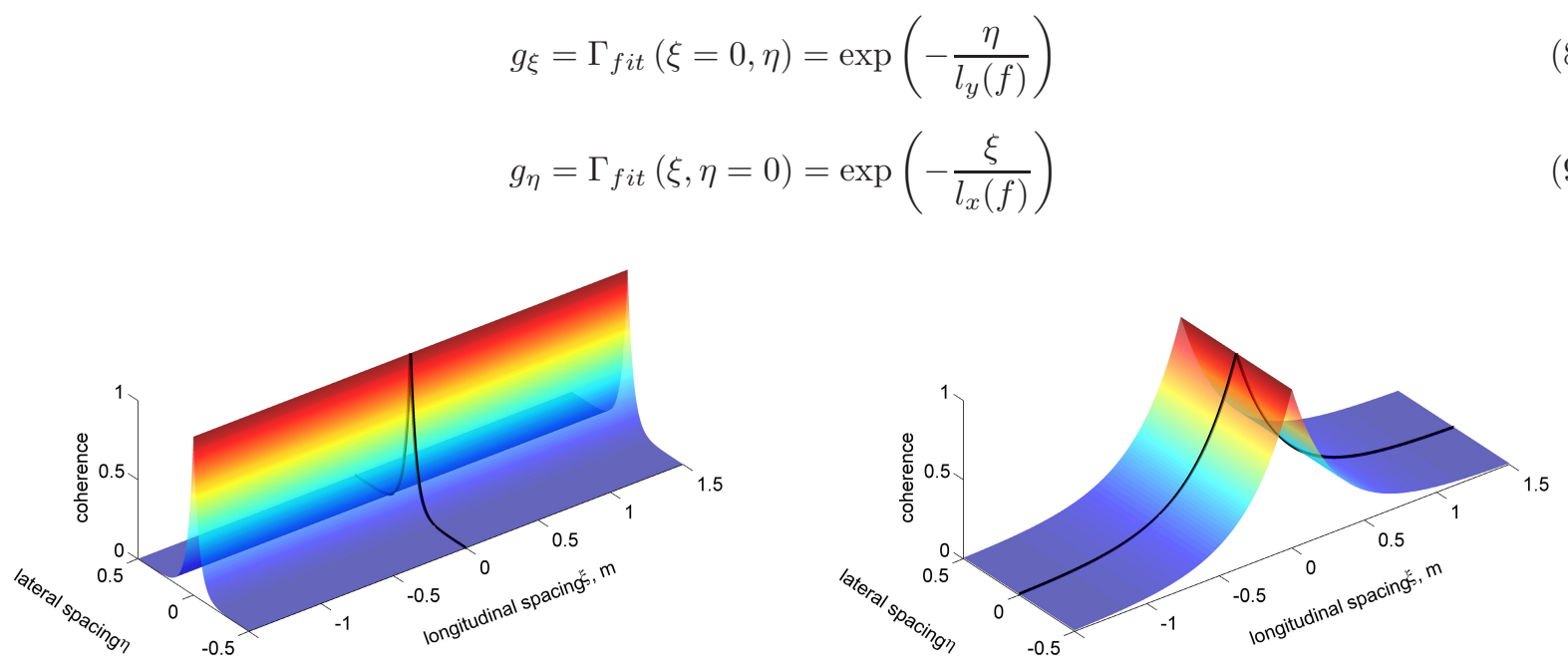

Figure 4. weighting factor for the longitudinal separation: lateral coherence decay (exemplary: $l_{y}=0.05 \mathrm{~m}$ )

Figure 5. weighting factor for the lateral separation: longitudinal coherence decay (exemplary: $l_{x}=0.3 \mathrm{~m}$ )

\section{Results}

The Efimtsov fit described by equation (4) through the points extracted by the analysis is shown in figure 6 for FL350 and TAS $=213 \mathrm{~m} \mathrm{~s}^{-1}$. The Efimtsov trial function provides a very good match with the experimental data points for the coherence length, when the coefficients are adapted to the present case (table 2). As expected, the coherence length has a high value at low frequencies and shows decay with frequency. A length limitation at low frequency is visible at $f \approx 300 \mathrm{~Hz}$ which is caused by a turbulent boundary layer structure size limitation. The goodness of the exponential fit is depicted as black error bars in this figure. The deviation is considerably low above a frequency of $1500 \mathrm{~Hz}$. Below $1500 \mathrm{~Hz}$, the deviation of the coherence decay data from an exponential shape increases.

\begin{tabular}{|l|c|c|c|c|c|c|}
\hline & $a_{1}$ & $a_{2}$ & $a_{3}$ & $a_{4}$ & $a_{5}$ & $a_{6}$ \\
\hline Efimtsov original & 0.1 & 72.8 & 1.54 & 0.77 & 548 & 13.5 \\
Corcos original & 0.1118 & 0 & - & 0.6656 & 0 & - \\
\hline
\end{tabular}

Table 2. Efimtsov Parameters

A comparison of the measured values with the predicted coherence lengths from the original Efimtsov model and the Corcos model at the same flight conditions is shown in figure 6 . Considerably higher values are measured in the frequency range below $1 \mathrm{kHz}$ than predicted by the Efimtsov model. The considerably 
lower maximum of the predicted coherence length value shows a difference in the limitation due to the boundary layer thickness. Higher coherence length values in the low-frequency range might be caused by a greater portion of low-frequency structures with large sizes in the boundary layer. Above $1.0 \mathrm{kHz}$, the Efimtsov-predicted and the measured value coincide well and approach further with increasing frequency. The coherence length values as predicted by the Corcos model coincide well at frequencies above $500 \mathrm{~Hz}$. Below, this frequency, the Corcos model dramatically overestimates the coherence length values due to its lack of size limitation.

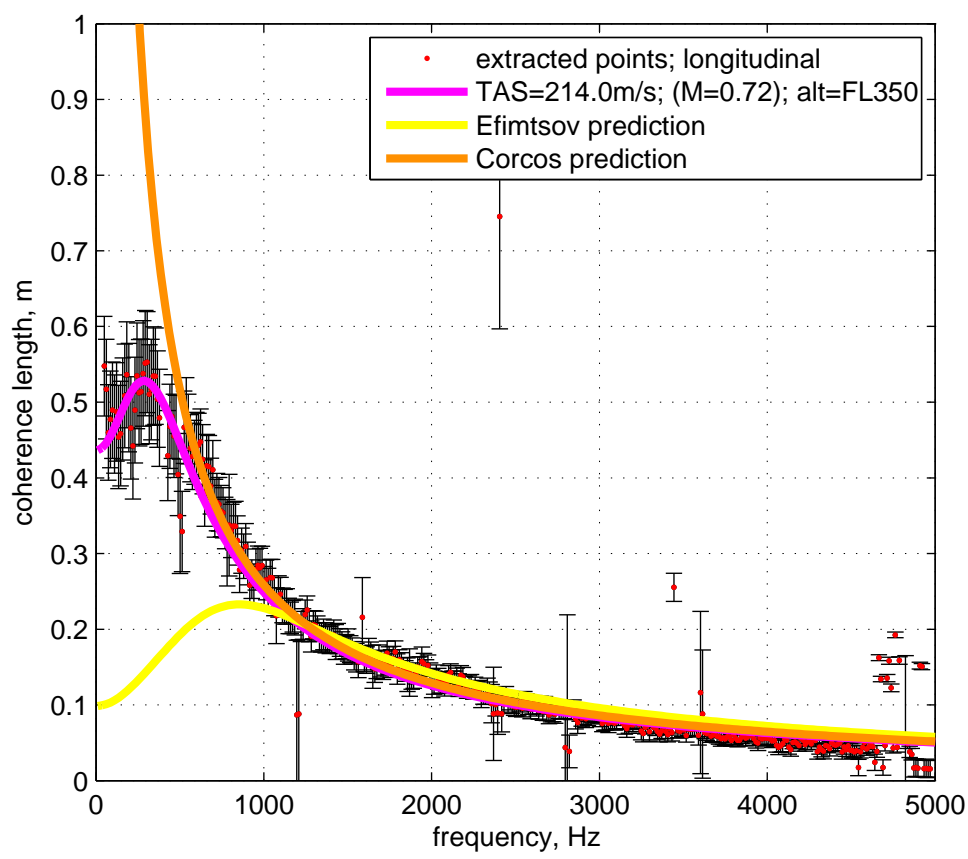

Figure 6. Coherence length model extracted from data points at FL350 and TAS=214m/s - longitudinal

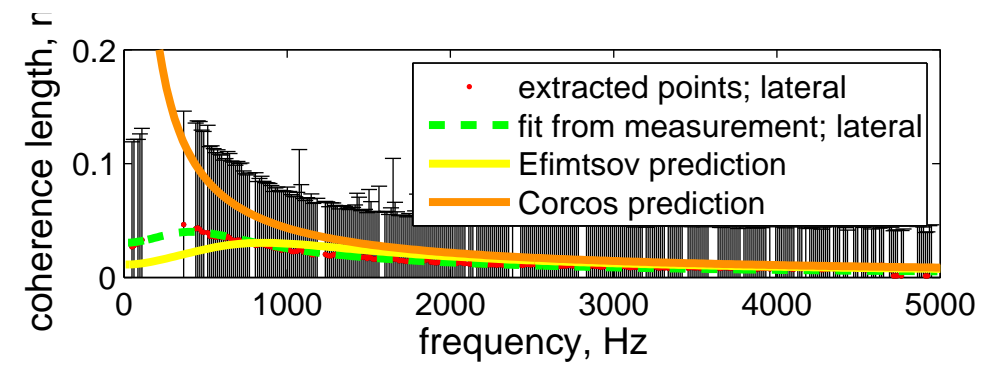

Figure 7. Coherence length model extracted from data points FL350 and TAS=214 m/s - lateral

A relatively high deviation level relative to the measured values can be seen for the lateral coherence length in figure 7. Nevertheless, the curve described by equation (4) with lateral coefficients approximates the measurement very well. A maximum can be found at a frequency of approximately $300 \mathrm{~Hz}$ in the same region as the maximum occurs in the longitudinal curve in figure 6 . The yellow Efimtsov-predicted curve shows a comparable maximum value. However, this peak is very broad and occurs at a higher frequency of $900 \mathrm{~Hz}$. The orange Corcos-predicted curve shows a good match of lateral coherence length above a frequency of $1000 \mathrm{~Hz}$. Below, it overestimates the lateral coherence length. On the whole, the value for the lateral coherence length is lower than the analysis deviation shown by the black bars. The further investigation will concentrate on the longitudinal coherence length. 


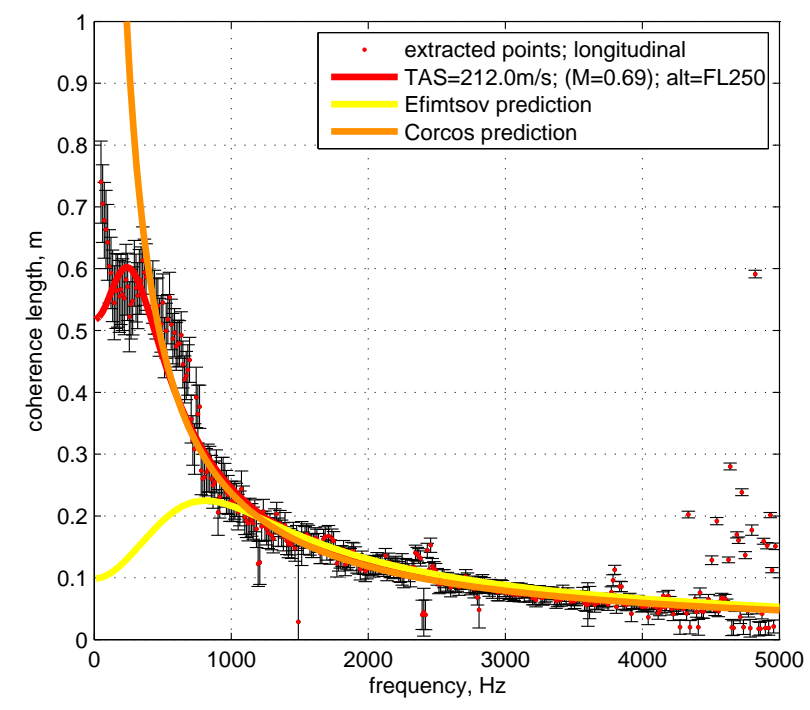

Figure 8. Coherence lengths for FL250; TAS $=212.0$ $\mathrm{m} / \mathrm{s}$

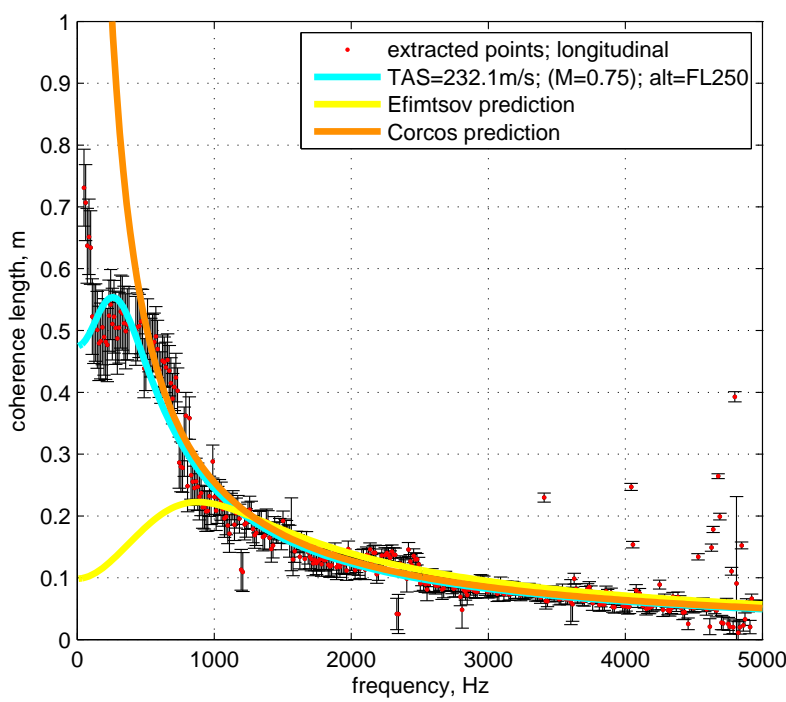

Figure 10. Coherence lengths for FL250; TAS $=232.1$ $\mathrm{m} / \mathrm{s}$

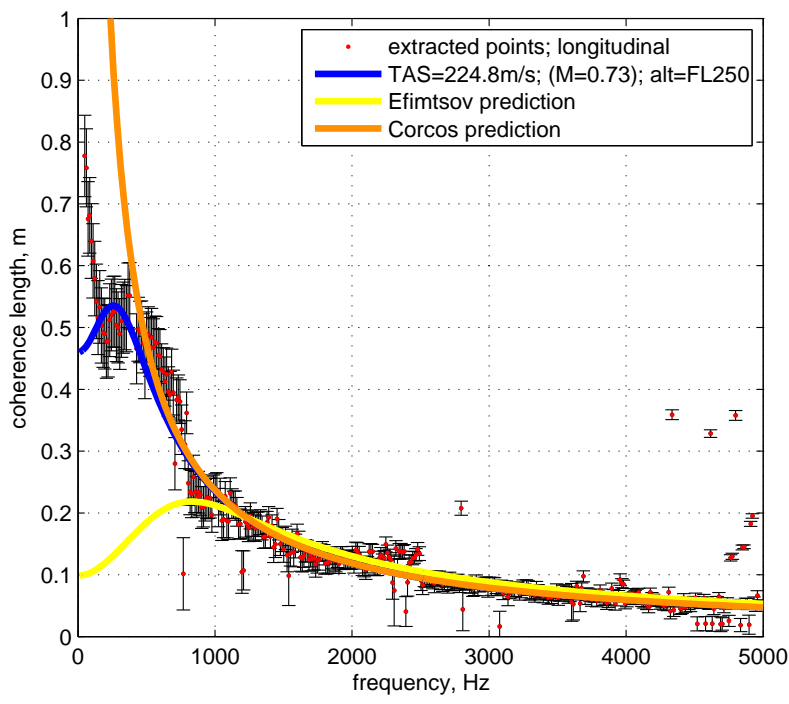

Figure 9. Coherence lengths for FL250; TAS $=224.8$ $\mathrm{m} / \mathrm{s}$

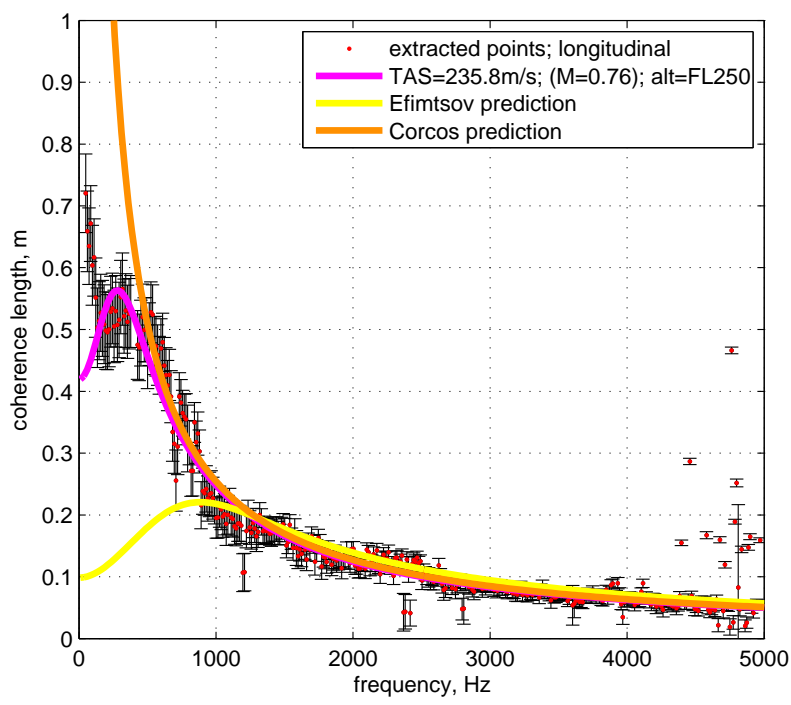

Figure 11. Coherence lengths for FL250; TAS $=235.8$ m/ 


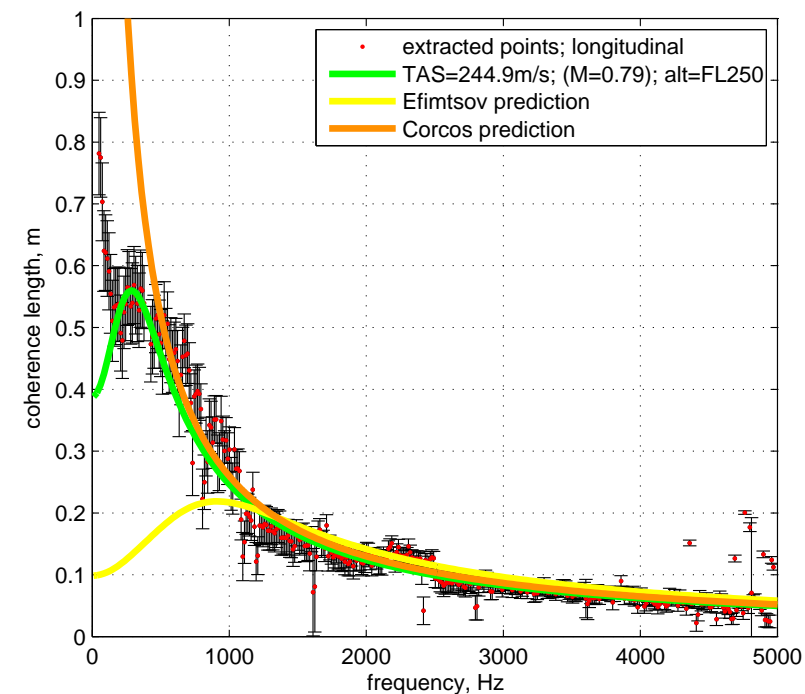

Figure 12. Coherence lengths for FL250; TAS $=244.9$ $\mathrm{m} / \mathrm{s}$

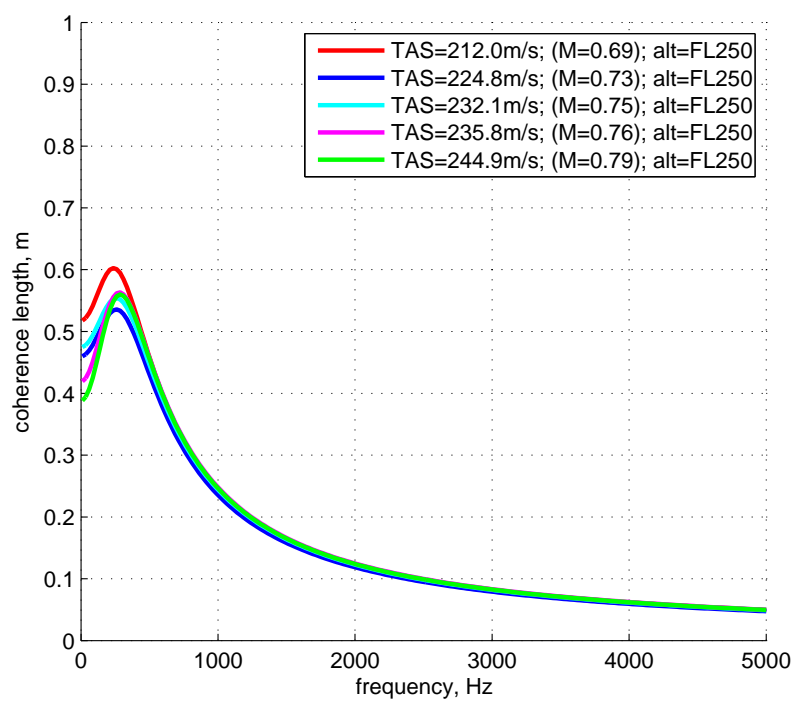

Figure 13. Characteristic of the longitudinal coherence length with variation of flight speed

\section{A. Influence of flow velocity}

The flow velocity was varied during the flight as described in the first column of table 1 . The measured coherence lengths with their fitted curves are shown in figures 8 - 12 together with the Efimtsov- and Corcospredictions for the same flight conditions. For all cases of the variation of flight speed, the Efimtsov model underestimates the maximum coherence length below $1000 \mathrm{~Hz}$. The Corcos model shows a good match of the measured data above $500 \mathrm{~Hz}$. Below, it clearly overestimates the measured values.

The fitted curve approximates the measured coherence length values very well. The boundary layer limitation is resolved good in the frequency range from $200 \mathrm{~Hz}$ up to $600 \mathrm{~Hz}$. Below $200 \mathrm{~Hz}$, a steep increase of the coherence length value can be seen for all the flight attitudes, which is not resolved by the fit.

A summary of the fitted curves for different flight speeds is shown in figure 13. The lines coincide very well above a frequency of $500 \mathrm{~Hz}$. Below this frequency, the lines show a little spread. The maximum coherence length for all speeds except the slowest lies at $0.55 \mathrm{~m}$ at a frequency of $f \approx 250 \mathrm{~Hz}$. The lowest flight speed stands out a little with a slightly higher peak values of $0.6 \mathrm{~m}$ at the same frequency. All in all, the coherence length characteristic seems to be constant with flight level.

Figure 14 shows a summary of just the deviation of the measured coherence decay from an exponential behavior (equation (7)). At high frequencies, this deviation has a constant level of $\sigma_{\text {fit }} \approx 0.01 \mathrm{~m}$. At low frequencies, the deviation increases linearly to a maximum value of $\sigma_{f i t} \approx 0.06 \mathrm{~m}$. The slope of the increase seems to be dependent of flight speed, leading to different deviation levels for different speeds in this area.

The level of the deviation is shown for a variation of flight speed over the Strouhal number formed with inner variables in figure 15 and with the Strouhal number formed with outer variables in figure 16. A good overall collapse of the curves is seen in both figures.

\section{B. Influence of flight altitude}

The flight altitude was varied as summarized in the first row of table 1. Figures 17 - 21 depict the measured coherence lengths along with the predicted curves resulting from the original Efimtsov model and the Corcos model. The deviation level for the respective flight test case is depicted as black bars. Above a frequency of $f=1000 \mathrm{~Hz}$ the values predicted by the Efimtsov model and the measured coherence lengths match very well. Below $1000 \mathrm{~Hz}$ the measured coherence length values exceed the Efimtsov prediction. The predicted values of the Corcos model match the measured values very well above a frequency of approximately $500 \mathrm{~Hz}$. Below this frequency, the Corcos model again overestimates the measured values due to its lack of boundary layer limitation.

The fitted curves resolve the measured points very well at high altitudes. A distinct peak as a limitation 


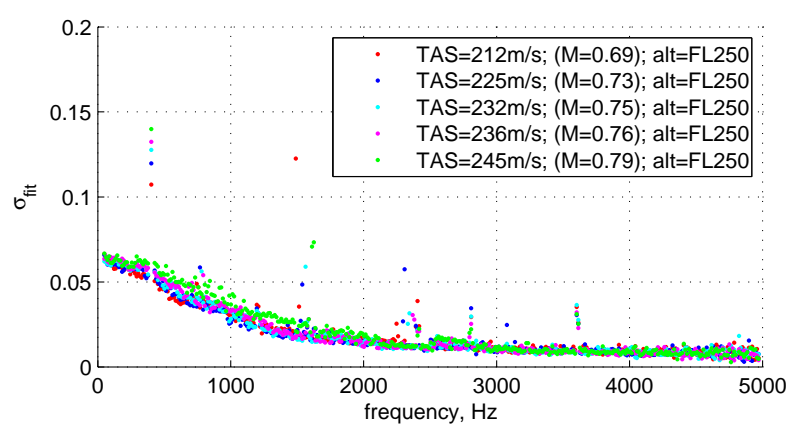

Figure 14. Deviation of the exponential fit to the measured coherence data, variation of speed; frequency scaling

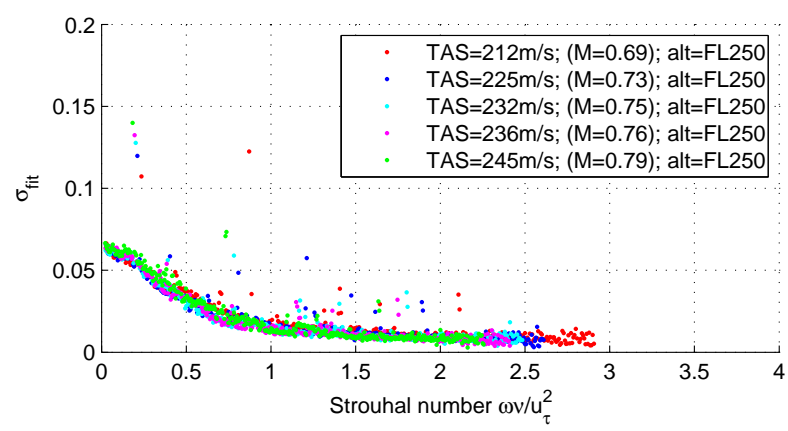

Figure 15. Deviation of the exponential fit to the measured coherence data, variation of speed; Strouhalscaling on inner variables

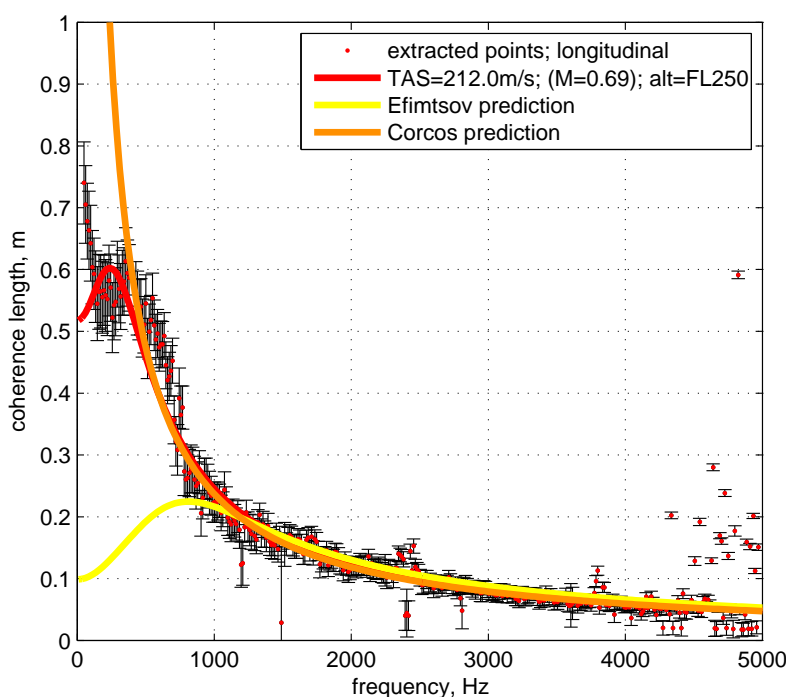

Figure 17. Coherence lengths for FL250; TAS $=212.0$ $\mathrm{m} / \mathrm{s}$

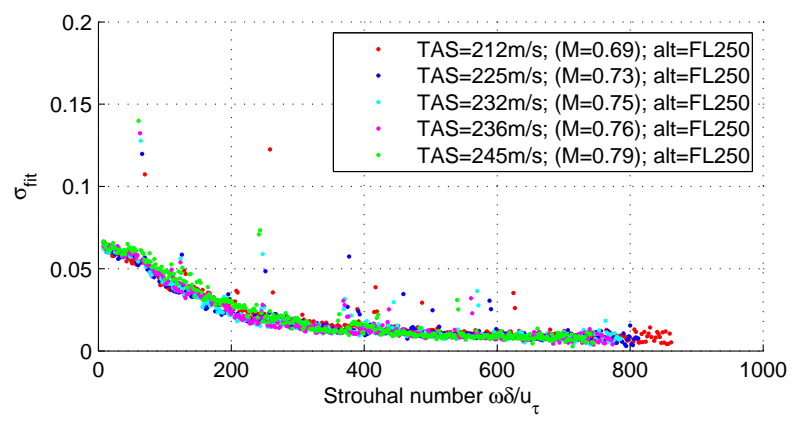

Figure 16. Deviation of the exponential fit to the measured coherence data, variation of speed; Strouhalscaling on outer variables

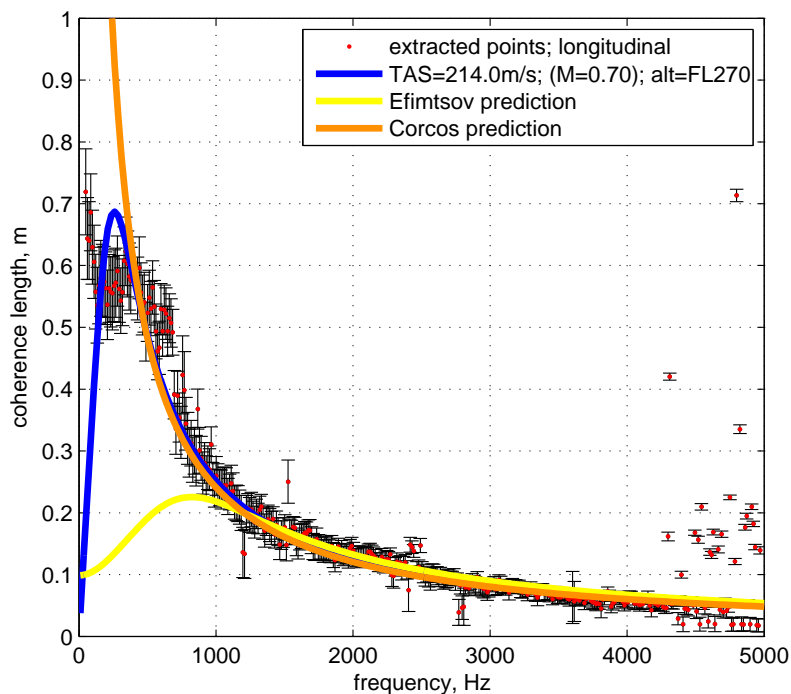

Figure 18. Coherence lengths for FL270; TAS $=214.0$ $\mathrm{m} / \mathrm{s}$ 


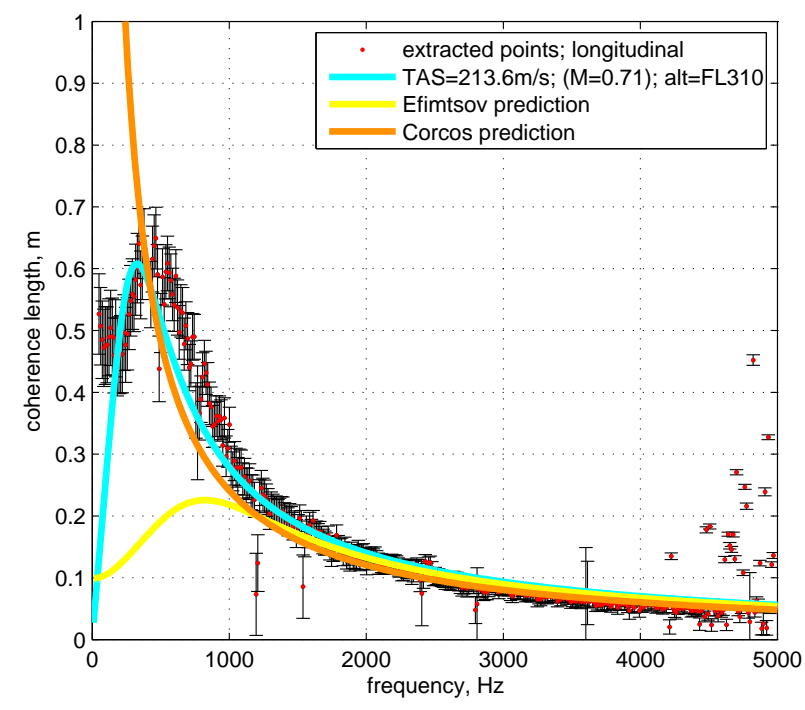

Figure 19. Coherence lengths for FL310; TAS $=213.6$ $\mathrm{m} / \mathrm{s}$

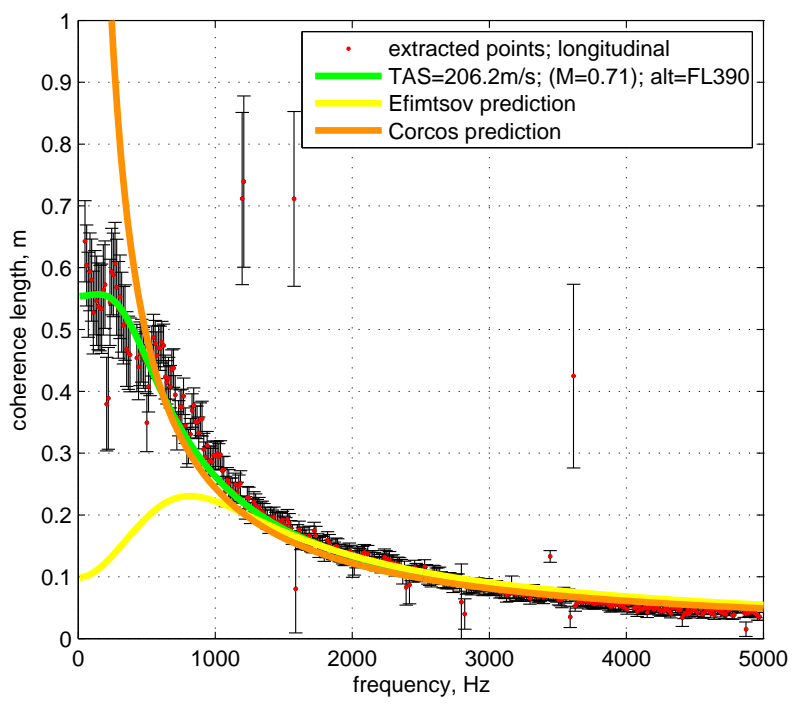

Figure 21. Coherence lengths for FL390; TAS $=206.2$ $\mathrm{m} / \mathrm{s}$

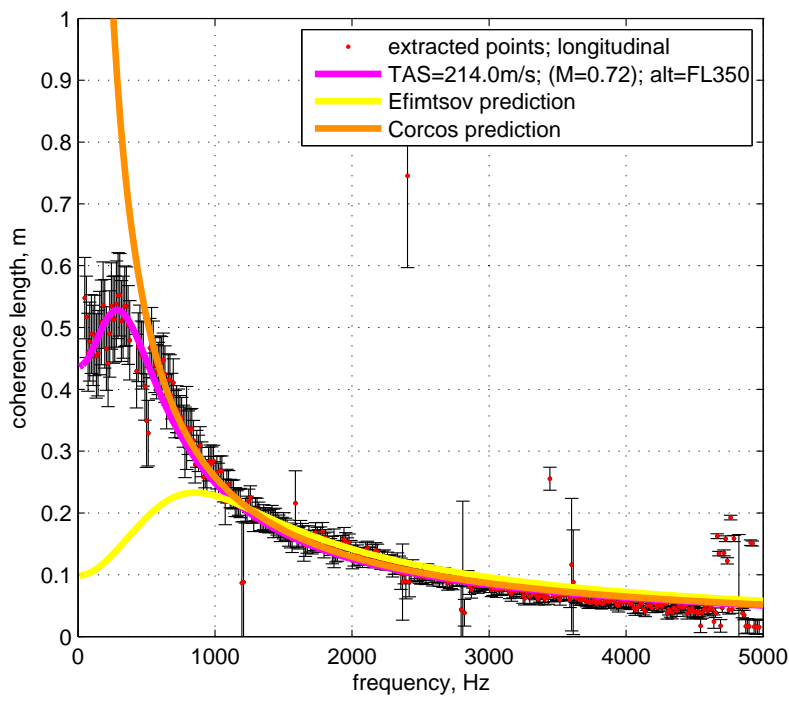

Figure 20. Coherence lengths for FL350; TAS $=214.0$ $\mathrm{m} / \mathrm{s}$

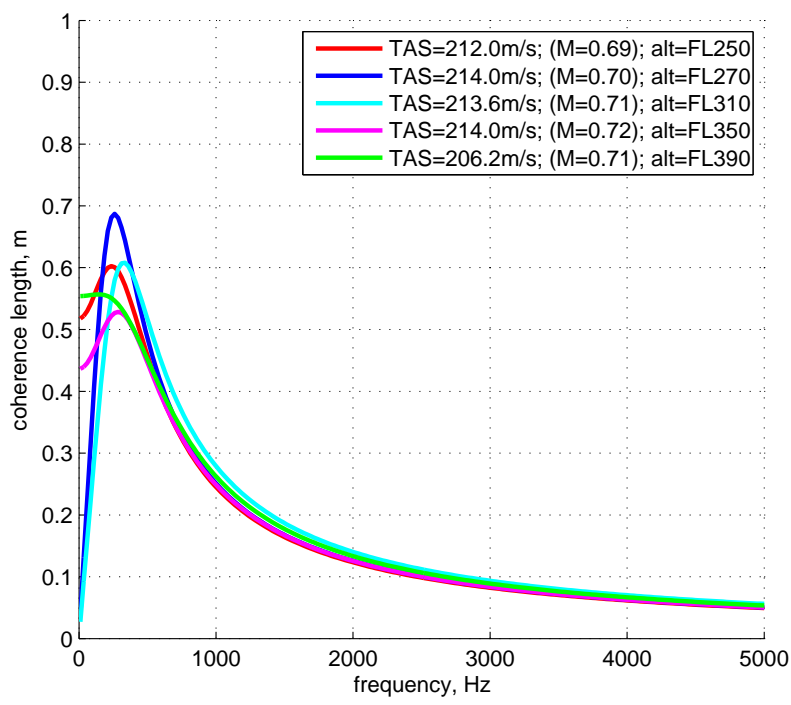

Figure 22. Characteristic of the longitudinal coherence length with variation of flight altitude 
of coherence length is found in the measurement points between $200 \mathrm{~Hz}$ and $600 \mathrm{~Hz}$, except for the highest altitude of FL390. The lowest two altitudes of FL250 and FL 270 exert a very steep increase of coherence length below $f \approx 200 \mathrm{~Hz}$. This steep rise of coherence length seems to disappear with increasing altitude.

The maximum coherence length due to the boundary layer limitation seems to decrease with flight altitude. In the low-frequency region, where the limitation occurs, flight levels 390 and 350 have a tendency to a lower maximum coherence length than the other three flight conditions. The curve of FL270 stands out of this. However, figure 18 shows, that this fit overshoots the measurement points slightly.

A summary of the fitted curves for the variation of flight altitude is shown in figure 22. Again, the curves show no considerable change of the characteristic for variation of altitude above a frequency of $600 \mathrm{~Hz}$. Below this frequency, the curves vary a considerable amount due to the increase of the measurement deviation.

Figure 23 shows the deviation of the coherence decay from exponential behavior for all measurements concerning the variation of flight altitude over frequency. Again, a constantly low deviation level is observed at high frequency. In other frequency ranges, the deviation shows a considerable variation with change of altitude. With decreasing frequency, the deviation increases up to a peak value. The maximum level of this peak is dependent on the flight altitude: the higher the altitude, the higher the peak at low frequency. In the mid-frequency range from $500 \mathrm{~Hz}$ to $1000 \mathrm{~Hz}$, the opposite effect can be observed: the higher the altitude, lower the deviation at mid-frequency.

When plotted over the Strouhal number formed with characteristic inner variables (figure 24) and with characteristic outer variables (figure 25) a good collapse can be observed in different areas of the plot. Using inner variables leads to a slightly better collapse of the data above $S h_{i n}=0.5$ in figure 24 . Using outer variables leads to a good collapse of the peak position at low Strouhal number (figure 25).

The deviation of the coherence decay from exponential behavior with variation of flight altitude can be assumed to have a cause at a macroscopic scale in the order of boundary layer size for $\omega \delta / u_{\tau}<=100$. In contrast, the deviation can be assumed to have a cause at a rather microscopic scale for $0.5<\omega \delta / u_{\tau}<2$.

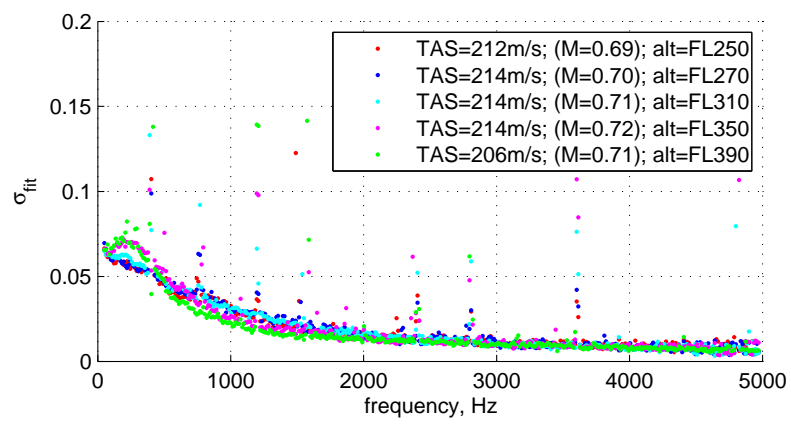

Figure 23. Deviation of the exponential fit to the measured coherence data, variation of altitude; frequency scaling

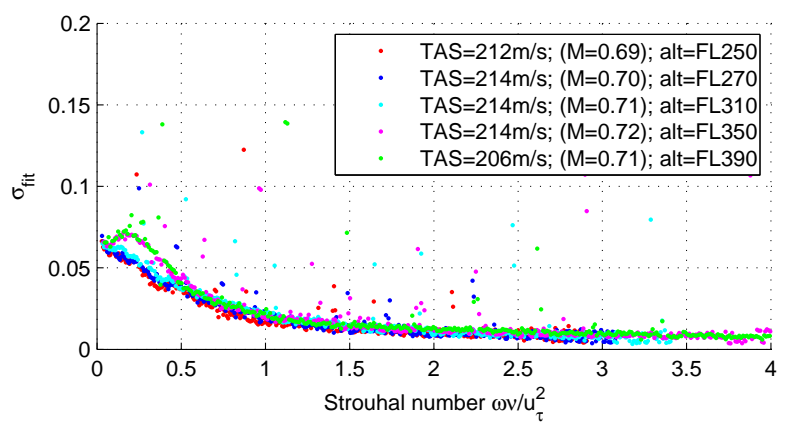

Figure 24. Deviation of the exponential fit to the measured coherence data, variation of altitude; Strouhalscaling on inner variables

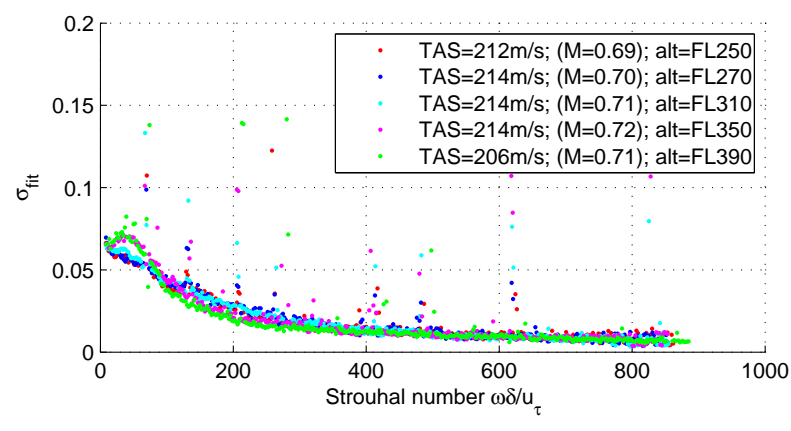

Figure 25. Deviation of the exponential fit to the measured coherence data, variation of altitude; Strouhalscaling on outer variables 


\section{Influence of both, flow velocity and flight altitude}

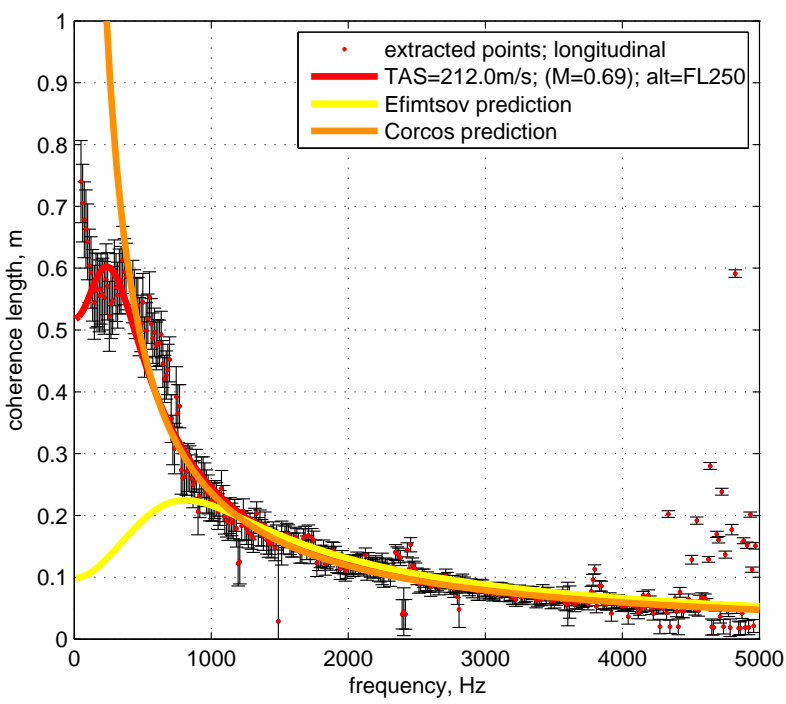

Figure 26. Coherence lengths for FL250; TAS $=212.0$ $\mathrm{m} / \mathrm{s}$

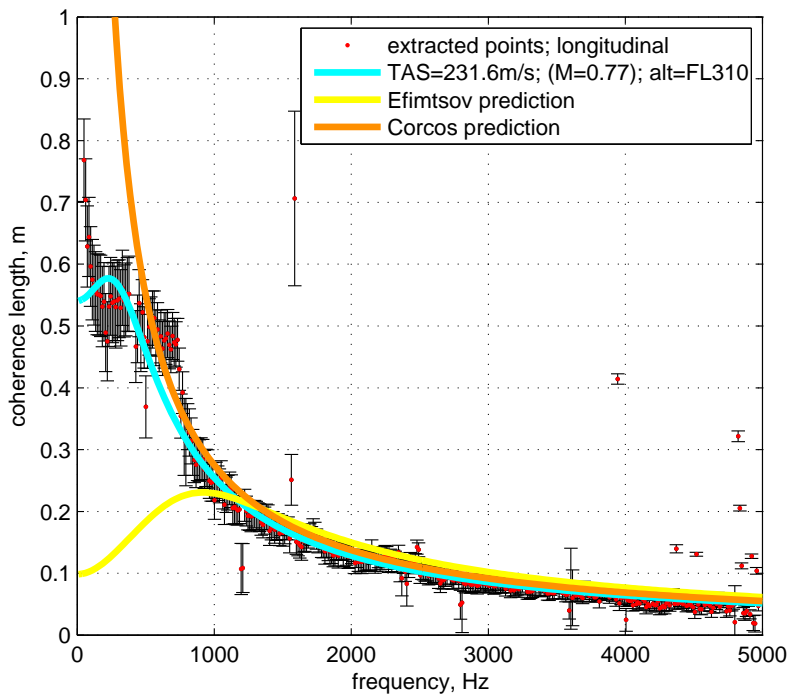

Figure 28. Coherence lengths for FL310; TAS $=231.6$ $\mathrm{m} / \mathrm{s}$

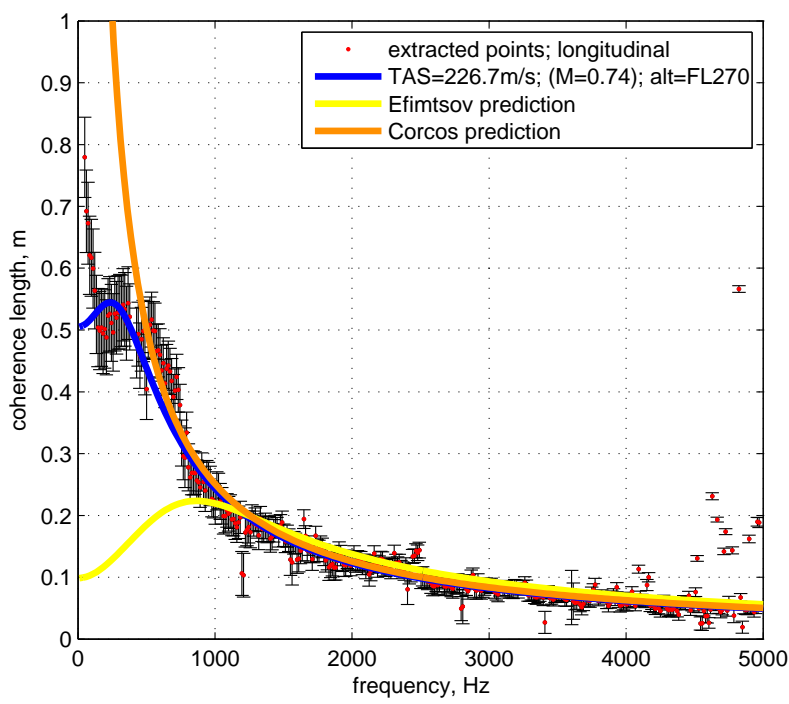

Figure 27. Coherence lengths for FL270; TAS $=226.7$ $\mathrm{m} / \mathrm{s}$

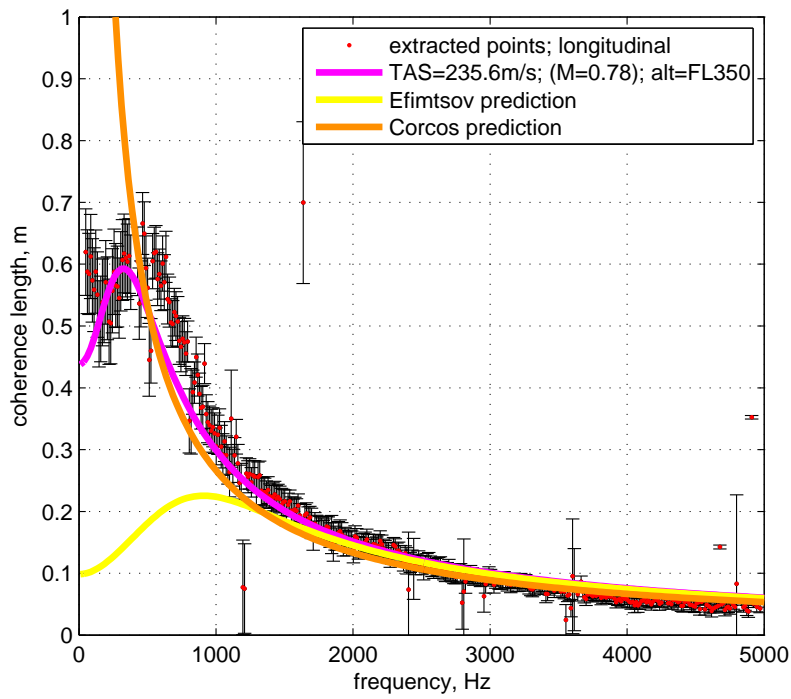

Figure 29. Coherence lengths for FL350; TAS $=235.8$ $\mathrm{m} / \mathrm{s}$

Flow velocity and flight altitude were varied together as summarized diagonally in table 1 . Plots of each single flight condition are shown in figures 26 - 30. Again, the Efimtsov model underestimates the measured values below $1000 \mathrm{~Hz}$ and the Corcos model overestimates the measured values below $500 \mathrm{~Hz}$. Above these frequencies, the models coincide with the measured values very well.

Again, the fitted curve with adapted parameters matches the behavior of the coherence length very good. The peak at low frequency is resolved well. At frequencies below $f \approx 200 \mathrm{~Hz}$, a very steep increase of coherence length is observed at the lower altitudes. The increase disappears again at higher altitudes. Contrary to the separate variation of altitude alone, the steep increase at low frequency is still present at FL310.

The combination of a variation of flight speed and altitude shows a change in the variation of the longitudinal coherence length (figure 31). The five curves are grouped into two characteristics: The altitude/speed- 


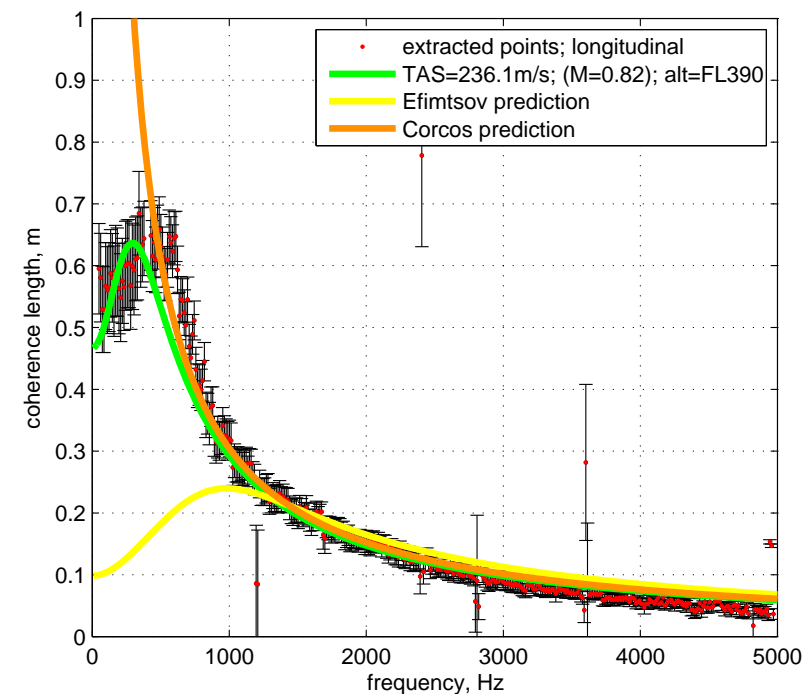

Figure 30. Coherence lengths for FL390; TAS $=236.1$ $\mathrm{m} / \mathrm{s}$

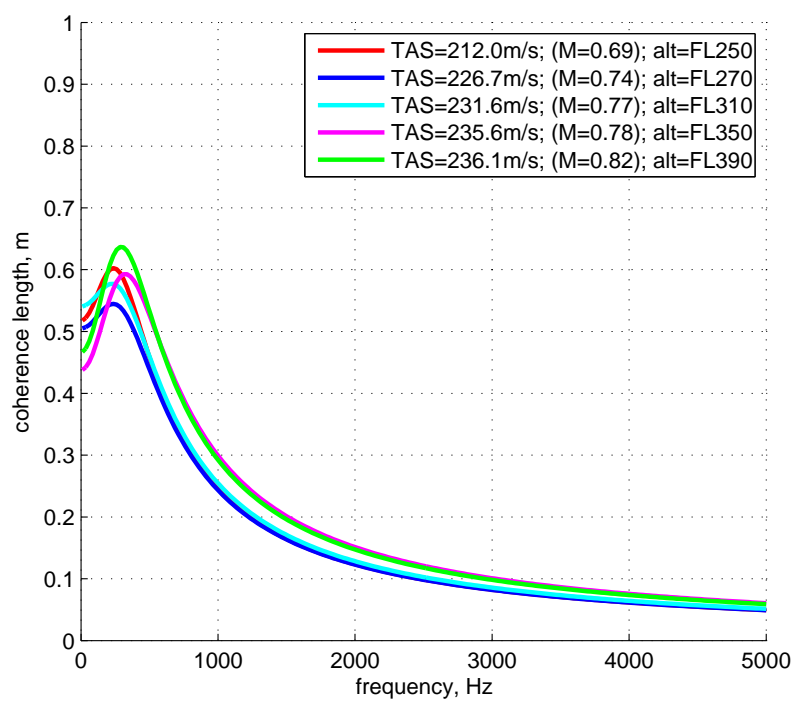

Figure 31. Characteristic of the longitudinal coherence length with variation of flight speed \& altitude

combinations below FL310 and $231.6 \mathrm{~m} \mathrm{~s}^{-1}$ (cyan, blue, and red curve) coincide above a frequency of $500 \mathrm{~Hz}$. The two remaining flight attitudes (magenta and green curve) coincide as well above $500 \mathrm{~Hz}$, yet they show a considerably higher coherence length than the first three curves up to $4000 \mathrm{~Hz}$. The two deviant curves (FL350 \& FL390) were recorded each on a separate day. Only the temperature of the measurement at FL350 stands out in figure 2. So far, no conclusive explanation was found for these findings.

Figure 32 shows the deviation of the coherence decay from exponential behavior for variation of both flight altitude and speed. At low frequency, the variation of the peak maximum level can be seen as from the variation of flight altitude independently. Besides this, the curves are aligned very well; the decrease of the deviation in the mid-frequency range is not observed.

When plotted over the Strouhal numbers formed inner variables (figure 33), the data points and the peak position from the different measurements do not collapse, although one would have expected it from the individual variation of altitude in figure 24. When using outer variables for the formation of the Strouhal number, the curves and the position of the peak coincide much better (figure 34). Surprisingly, the overall collapse is good as well.

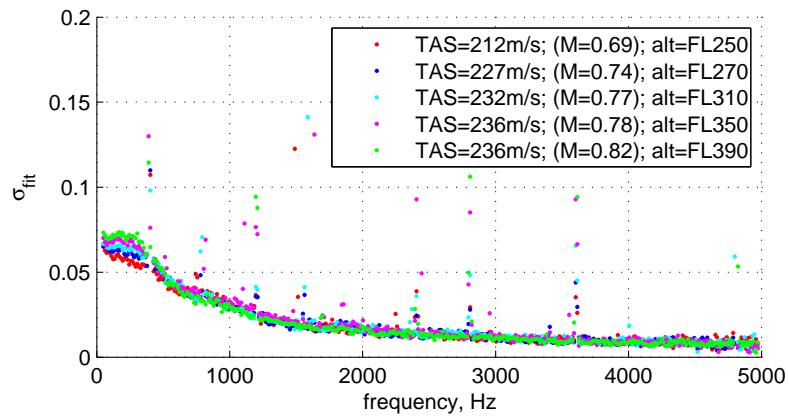

Figure 32. Deviation of the exponential fit to the measured coherence data, variation of both altitude \& speed; frequency scaling

\section{Conclusion \& Outlook}

The coherence length within a turbulent boundary layer was measured at a fixed position on the aircraft in flight at different speeds and altitudes. A considerable increase of coherence length compared to the 


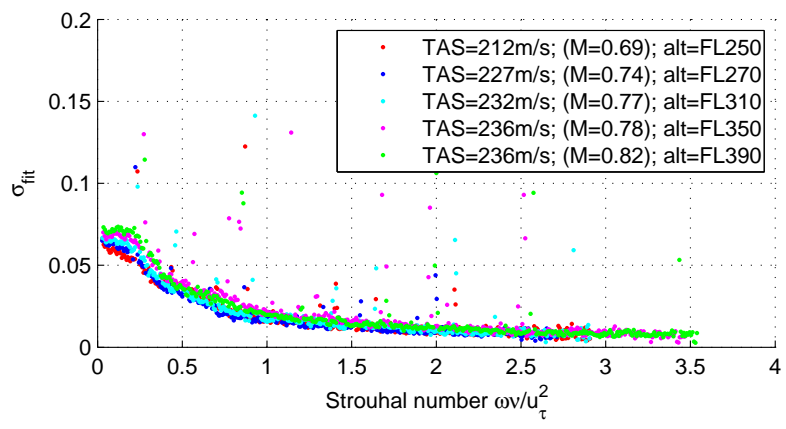

Figure 33. Deviation of the exponential fit to the measured coherence data, variation of both altitude \& speed; Strouhal-scaling on inner variables

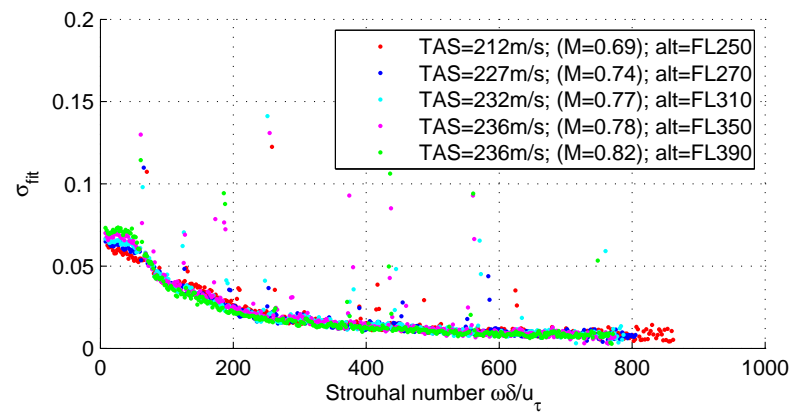

Figure 34. Deviation of the exponential fit to the measured coherence data, variation of both altitude \& speed; Strouhal-scaling on outer variables

prediction of Efimtsov is visible in the low-frequency range below $1 \mathrm{kHz}$ as was found by others. ${ }^{4}$ Above this frequency, the Efimtsov prediction matches the measured data very good. The Corcos model was suitable above a frequency of $500 \mathrm{~Hz}$. Below, it dramatically overestimates the coherence length values due to its lack of size limitation. The fit function introduced by Efimtsov was used to parameterize the observed behavior. It was found that the function will describe the behavior of coherent structures within the turbulent boundary layer very good, if the parameters for the boundary layer limitation are adapted. The limitation of coherence length at low frequencies has been introduced by Efimtsov to account for the limitation of turbulent structure size by the boundary layer thickness. Considerably larger turbulent structures are assumed to occur in the boundary layers of the present test cases. An investigation of the influence of boundary layer thickness at different positions on the aircraft may lead to a better ability to adapt the Efimtsov parameters.

When varying the flight speed at constant altitude, no effects were seen in the characteristic of the coherence length behavior. When varying the altitude at constant speed, two changes in the characteristic were observed in the low-frequency range. First, the level of the boundary layer limitation dropped to a slightly lower maximum coherence length value with increasing altitude. Secondly, at very low frequency below the limitation, a very steep increase in coherence length was visible at lower altitudes, which disappeared gradually with increasing flight level.

The joint variation of both flight speed and altitude showed an increase of coherence length at the two flight attitudes with the highest altitude and the highest speed. This increase cannot be explained by the variation of altitude and speed separately.

Besides the coherence length, also the deviation of the measured coherence decay from exponential behavior was investigated. Considerable deviation is observed in the low frequency region. By collapsing it using different characteristic variables for the formation of the Strouhal number, the cause for this deviation was is believed to be present at a macroscopic scale in the order of the boundary layer thickness. An appropriate fit function for the frequency range where the higher deviation occurs can be determined in a further evaluation of the present data. It could lead to a better understanding of the decay of the coherence of turbulent structures in the boundary layer at high subsonic Mach numbers and flight-relevant altitudes.

\section{Acknowledgements}

The authors would like to thank the following institutions for their contribution:

- The Federal Ministry of Economics and Technology (BMWi) for the financial support of "SIMKAB" (Simplified Cabin) as part of the aerospace research program (LuFO IV).

- Airbus S.A.S. for the provided infrastructure and help on modifications of the test carrier.

- The DLR Institute of Aeroelasticity, DLR Institute of Flight Systems, and the DLR Flight Experiment team for the help provided in the preparation and carry-out of the flight test. 


\section{References}

${ }^{1}$ Efimtsov, M., "Characteristics of the Field of Turbulent Pressures at the Wall of a Boundary Layer," Sov. Phys. Acoust., Vol. 28, No. 4, 1982, pp. 289-292.

${ }^{2}$ Corcos, G. M., "Resolution of Pressure in Turbulence," The Journal of the Acoustical Society of America, Vol. 35, No. 2, 1963, pp. 192-199.

${ }^{3}$ Graham, W., "A Comparison of Models for the Wavenumber-Frequency Spectrum of Turbulent Boundary Layer Pressures," Journal of Sound and Vibration, Vol. 206, No. 4, 1997, pp. 541-565.

${ }^{4}$ Palumbo, D. L., "Measurement of the Correlation and Coherence Lengths in Boundary Layer Flight Data," Tech. Rep. TM-2011-217060, NASA, 2011.

${ }^{5}$ Spehr, C., Hennings, H., Bouhaj, M., Haxter, S., Hebler, A., and Buchholz, H., "In-flight sound Measurements: A first overview," Submitted for publication, 18th AIAA/CEAS Aeroacoustics Conference, Colorado Springs, Colorado, AIAA, June 2012.

${ }^{6}$ Haxter, S. and Spehr, C., "Two-Dimensional Evaluation of Turbulent Boundary Layer Pressure Fluctuations at Cruise Flight Condition," Submitted for publication, 18th AIAA/CEAS Aeroacoustics Conference, Colorado Springs, Colorado, AIAA, June 2012.

${ }^{7}$ Bies, D. A., "A Review of Flight and Wind Tunnel Measurements of boundary Layer Pressure Fluctuations and Induced Structural Response," Tech. Rep. 29134, NASA, 1966.

${ }^{8}$ Gyorgyfalvy, D., "Effect of Pressurization on Airplane Fuselage Drag," Journal of Aircraft, Vol. 2, No. 6, 1965 , pp. 531-537.

${ }^{9}$ Haxter, S., Ehrenfried, K., and Kröber, S., "Examination of the influence of flow speed on the coherence lengths in turbulent boundary layers at high subsonic Mach numbers," Notes on Numerical Fluid Mechanics and Multidisciplinary Design, Submitted for publication. 